\title{
Fibrin sealant use in thyroidectomy: A prospective, randomized, placebo-controlled double blind trial using EVICEL
}

\author{
Joshua D. Hornig, M. Boyd Gillespie, Eric J. Lentsch, Colin W. Fuller, Jackson Condrey and Shaun A. Nguyen* \\ Department of Otolaryngology-Head and Neck Surgery, Medical University of South Carolina, Charleston, SC, USA
}

\begin{abstract}
Objectives: To evaluate EVICEL fibrin sealant used in thyroid surgery closure. Primary endpoints were post-operative drain output, time to drain removal, length of admission and adverse events.

Study design: A prospective, randomized, double-blind, placebo-controlled study.

Settings: Subjects seen in the ENT Clinic at the Medical University of South Carolina.

Subjects and methods: From June 2010 to January 2014, an IRB-approved prospective, randomized, double-blind study of EVICEL versus a saline control was conducted on 70 subjects receiving total thyroidectomy or hemithyroidectomy. 28 received Evicel and 27 received saline; data from fifteen subjects were eliminated due to protocol violations. The mean age was 50.3 (range 21 to 73 ).

Results: There was no significant difference in drain output between Evicel (median[interquartile range]: $96.3 \mathrm{~mL}$ [73.3-139.3 mL]) and placebo (120.0 mL [68.8$161.5 \mathrm{~mL}$ ], p=0.334). Drain time (37.9 hrs [25.2-48.7 hrs] vs. $43.6 \mathrm{hrs}$ [37.6-58.1 hrs]) and hospital stay (45.5 hrs[33.4-53.8 hrs] vs. 50.9 hrs[44.1-69.4 hrs]) were also shorter for Evicel, but again these differences were not significant ( $\mathrm{p}=0.101$ and 0.526 respectively). For total thyroidectomy subgroup there was a significant reduction in drain output $(103.5 \mathrm{~mL}$ [80.0-138.6 mL] vs. $150.0 \mathrm{~mL}[120.0-188.5 \mathrm{~mL}$ ], p=0.035) and drain time (40.3 hrs [26.2-49.1 hrs] vs. 47.1 hrs [42.0-67.8], $\mathrm{p}=0.035$ ) with Evicel. Hospital stay in this subgroup was shorter with Evicel (50.3 hrs [43.6-54.9 hrs] vs. 59.4hrs [48.4-70.6 hrs]), but this result was not significant $(\mathrm{p}=0.246)$. No outcomes were significant in the hemithryoidectomy subgroup. Nine adverse events occurred in the Evicel group compared to three for placebo $(\mathrm{p}=0.101)$.
\end{abstract}

Conclusion: Evicel sealant appears to be a safe, effective method to reduce serous drain output following total thyroidectomy, but has a limited role in hemithyroidectomy due to low levels of baseline drain output.

\section{Introduction}

Thyroid surgery is a relatively common procedure, owing in part to the high prevalence of thyroid nodules [1]. Although the morbidity and mortality of thyroid surgery is low, thyroidectomy is certainly not without risk. Due to the gland's proximity to the trachea and its rich blood supply, development of an airway-compressing hematoma remains the most dangerous post-operative complication of thyroid surgery. For this reason, thyroidectomy has traditionally included the use of drains during the post-operative period. In addition, the use of drains has been advocated to reduce the incidence of postoperative seroma formation, which may require serial aspiration or open drainage followed by compression dressings [2].

The routine use of drains, however, has recently been questioned. Since 2007, three separate meta-analyses [3-5] have shown no significant benefit to routine drainage, and in some cases clinicallysignificant detriment including increased risk of infection and prolongation of hospital stay compared to drain-free post-operative management. While individual studies have shown varied results regarding drains' ability to decrease seroma incidence, the most recent analysis has shown a non-significant trend toward reduction in seroma (risk ratio 0.38-1.09, $\mathrm{p}=0.1$ ) [4]. Postoperative hematoma is a sufficiently rare event, and its consequences sufficiently dire, that some practitioners remain hesitant to do away with the traditional approach
[6]. Additionally, the use of drains may still be indicated in select cases such as those with highly vascular glands (eg. Graves' disease); large or substernal goiters; and in cases combined with neck dissection [7].

Fibrin-based sealants have a long development history and were first approved by the United States Food and Drug Administration (FDA) in 1998. These products use a two-component system of liquidsuspended thrombin and fibrinogen, the final two molecules involved in the clotting cascade. When mixed, they produce a gelatinous fibrin clot that can be used as a biocompatible, resorptive sealant or glue. Fibrin sealants have been used in vascular, cardiac and trauma surgery for obtaining hemostasis when traditional methods are impractical or impossible [8-10]. Their application has been proposed and studied for several indications in otolaryngology [11-13], including as prophylaxis against hematoma in thyroid surgery. The present study is designed to determine the efficacy of Evicel fibrin sealant (Manufactured by Ethicon; Somerville, New Jersey, USA) to reduce post-thyroidectomy

Correspondence to: Dr. Shaun A. Nguyen, Associate Professor- Director of Clinical Research, Department of Otolaryngology-Head and Neck Surgery, Charleston, SC, USA, Tel: 843-792-1356; Fax: 843-792-0546, E-mail: nguyensh@musc.edu

Key words: fibrin sealant, thyroidectomy, hemithyroidectomy

Received: April 08, 2016; Accepted: May 07, 2016; Published: May 11, 2016 
wound drainage, drain time and hospital stay. The study design is a double-blind, randomized, placebo-controlled trial comparing Evicel fibrin sealant to saline placebo, using a traditional drainage protocol in total and partial thyroidectomy. While hematoma rates were recorded, the study was not designed with sufficient power to demonstrate a difference in this outcome; therefore, wound drainage was considered a proxy for the likelihood of developing hematoma and seroma.

\section{Methods}

Between June 2010 and December 2013, subjects undergoing total or partial thyroidectomy were consented for this industrysponsored prospective randomized placebo-controlled double-blind trial. The trial was approved by the Medical University of South Carolina (MUSC) Institutional Review Board (IRB). The costs of the trial covered by the sponsor (Ethicon; Somerville, New Jersey, USA) included complimentary product, patient costs not associated with the standard of care, institutional overhead and faculty percent effort, and salary support for a research study coordinator. The study was an investigator-initiated protocol written, executed, and analyzed solely by faculty and staff of the MUSC Department of OtolaryngologyHead \& Neck Surgery. Subjects were recruited from otolaryngology clinics at MUSC once they were scheduled for thyroid surgery. All subjects underwent thyroid surgery with one of four head and neck surgeons. In order to be enrolled, subjects had to satisfy the following inclusion criteria: initial diagnosis of neoplasm or goiter requiring surgical management; Eastern Cooperative Oncology Group (ECOG) performance status 0-2; no evidence of distant metastasis; no history of previous surgery or radiation therapy to the neck; no history of thyroid cancer or goiter recurrence; and no history of coagulation disorder. Planned or unplanned neck dissection or substernal goiter was not considered exclusion criteria for the study.

Subjects were randomized to placebo or Evicel group based on a computer-generated random number sequence. Study staff was paged to the operating room near the end of each procedure, and the senior surgeon stepped away from the surgical field. Evicel is a fibrin sealant stored in two components. For subjects in the treatment group, Evicel was applied in the usual manner by study personnel, with $5 \mathrm{~mL}$ of each component drawn into a two-barrel syringe device and aerosolized using a pedal-controlled inert gas supply. For subjects in the placebo group, saline was drawn into the applicator and aerosolized in a similar fashion; it was allowed to remain in the wound during closure. After instillation of Evicel or saline, either the junior surgeon completed the case or the strap muscles were closed to allow the senior surgeon to finish the procedure in a blinded fashion. At no point in the subjects' care were senior surgeons unblinded to the treatment group.

In every case, a $10 \mathrm{~mm}$ Jackson-Pratt drain was placed in the thyroid bed to siphon postoperative fluid, and subjects were managed postoperatively in the usual fashion. Drain output was recorded by blinded nursing staff every four hours postoperatively. When the drain output fell below $10 \mathrm{~mL}$ per eight hours, study staff removed the drain and the time of removal was recorded. Time from end of surgery to discharge was also recorded for analysis, as were adverse events.

\section{Statistical analysis}

Baseline characteristics of the subjects in the placebo and treatment groups were analyzed to determine the adequacy of randomization. Dichotomous variables, including gender and characteristics of surgery performed, were analyzed with chi-square tests. Age was analyzed with a student's t-test, while surgical specimen volume and mass were analyzed with Mann-Whitney U tests. Calculations were performed using QuickCalc statistical calculators available for free at Graphpad. com, except for the Mann-Whitney test, which was performed using the online calculator produced by faculty at Virginia Commonwealth University School of Medicine [14].

Outcome variables were analyzed for differences between placebo and treatment groups using a chi-square test for the occurrence of adverse events, and with Mann-Whitney U-tests for differences in drain output in the first eight postoperative hours, overall drain output, and time from end of surgery to discharge. The Mann-Whitney test was used due to the non-normal distributions of these variables. Additional analyses were performed on total thyroidectomy and hemithyroidectomy subgroups.

An alpha of 0.05 was used as the significance level for rejecting the null hypothesis in each test. To determine the desired population size we utilized data reported by Uweira et al. [15] to determine standard deviations for drainage volume at 8 hours post-op, total drainage volume, time to drain removal and time to discharge. These calculations showed a minimum sample size of 55 patients per group would be needed to demonstrate a difference of $20 \%$ between the groups at a power of $80 \%$. Allowing for the potential of in evaluable data on some patients, we came to the conclusion that a sample size of 60 would be appropriate for each group. However, the study was closed early due to low enrollment. Low enrollment may have been due in part to the fact that not all surgeons in our department were comfortable with the conservative drain management protocol required by the study.

\section{Results}

70 subjects were consented and enrolled. 55 subjects completed the study protocol and their data are included for analysis. Of these 55,27 were randomized to the treatment group and 28 to the control group (Table 1). 15 subjects were withdrawn from the study for various reasons, including: voluntary withdrawal by subject (6); intraoperative blinded withdrawal of subject by senior surgeon due to need to deviate from surgical protocol (such as the necessity of Avitene powder for hemostasis) (3); inadvertent unblinding of attending surgeon (2); study product on backorder (1); inappropriate Evicel application technique (1); staff oversight with premature completion of closure (1); and inadvertent early removal of drain by non-study staff (1). The 2010 CONSORT $^{16}$-compatible flow diagram is shown in Table 1 . Baseline characteristics of the 55 included subjects are shown in Table 1.

Table 2 displays the adverse events experienced by study subjects, categorized by treatment group and by total $v$ s. hemithyroidectomy. While more adverse events occurred in the treatment group, this difference was not statistically significant in the overall analysis or for any subgroup.

Overall treatment $v s$. placebo comparisons showed no significant difference between placebo and Evicel with regard to any study measure, including drain output during the first eight hours, total drain output, drain time, hospital stay and adverse events. The same held true for hemithyroidectomy subgroup comparison (Table 3a and $3 \mathrm{~b}$ ).

In the total thyroidectomy subgroup, there was a significant reduction in total drain output $(\mathrm{p}=0.035)$ and drain time $(\mathrm{p}=0.035)$ compared to placebo (Table $3 \mathrm{c}$ ). The lower drain output and shorter hospital stay in the Evicel group was not significant. Adverse events were more common with Evicel, but not significantly so $(\mathrm{p}=0.264)$. 
Table 1. Subject and procedure baseline characteristics.

\begin{tabular}{|c|c|c|c|c|c|}
\hline & & Placebo & Evicel & Test & Significance \\
\hline First 8hours output (mL) & Median & 45.0 (IQR: 28.8-74.0) & 50.0 (IQR: 34.3-70.3) & Mann-Whitney & 0.669 \\
\hline Total output $(\mathrm{mL})$ & Median & 120.0 (IQR: 68.8-161.5) & 96.3 (IQR: 73.3-139.3) & Mann-Whitney & 0.334 \\
\hline Drain time (hours) & Median & 43.6 (IQR: 37.6-58.1) & 37.9 (IQR: 25.2-48.7) & Mann-Whitney & 0.101 \\
\hline Hospital stay (hours) & Median & 50.9 (IQR: 44.1-69.4) & 44.9 (IQR: 32.3-53.2) & Mann-Whitney & 0.421 \\
\hline AEs & $\mathrm{n}, \%$ & $3,11.1 \%$ & $9,32.1 \%$ & Chi-square & 0.294 \\
\hline
\end{tabular}

Baseline characteristics of subjects entered into the trial. Dichotomouss variables were tested using Chi-square, while continuous variables were tested by t-test or Mann-Whitney U test, depending on the variable's normality.

(See statistical methods). Abbdevations: SD: standard deviation, $m L$ : Millilitrs, IQR: interquartile range, g- grams

Table 2. Adverse events.

\begin{tabular}{|l|l|l|}
\hline & Placebo & Evicel \\
\hline Total thyroiddectomy & $\begin{array}{l}\text { 2 cases of hypocalcemia below } 7.0 \mathrm{mg} / \mathrm{dL} \\
\text { I case of bilaterial TVC paralysis }\end{array}$ & $\begin{array}{l}1 \text { case of hypocalcemia below } 7.0 \mathrm{mg} / \mathrm{Dl} \\
\text { 2 additional cases of hypocalcemic tetany }(1 \mathrm{cotpatient}) \\
1 \text { case of laryngeal edema requiring overnight stay in ICU } \\
1 \text { non-admit ED visit for incisional and facial swelling } \\
1 \text { case of TVC paralysis requiring surgical correction } \\
1 \text { case of TVC paralysis persisting past } 6 \text { months }\end{array}$ \\
\hline Hemithyroidectomy & & $\begin{array}{l}1 \text { case of TVC paralysis requiring surgical correction } \\
1 \text { asymptomatic hematoma }\end{array}$ \\
\hline Total & 3 & 9 \\
\hline
\end{tabular}

Abbreviations: mg/dL: milligrams per deciliter, TVC: true vocal cord, ICU: intensive care unit, ED: emergency department

Table 3a. Overall results.

\begin{tabular}{|c|c|c|c|c|c|}
\hline & & Placebo & Evicel & Test & Significance \\
\hline First 8hours output (mL) & Median & 45.0 (IQR: 28.8-74.0) & 50.0 (IQR: $34.3-70.3$ ) & Mann-Whitney & 0.669 \\
\hline Total output $(\mathrm{mL})$ & Median & 120.0 (IQR: 68.8-161.5) & 96.3 (IQR: 73.3-139.3) & Mann-Whitney & 0.334 \\
\hline Drain time (hours) & Median & 43.6 (IQR: 37.6-58.1) & 37.9 (IQR: 25.2-48.7) & Mann-Whitney & 0.101 \\
\hline Hospital stay (hours) & Median & 50.9 (IQR: 44.1-69.4) & 44.9 (IQR: 32.3-53.2) & Mann-Whitney & 0.421 \\
\hline AEs & $\mathrm{n}, \%$ & $3,11.1 \%$ & $9,32.1 \%$ & Chi-square & 0.294 \\
\hline
\end{tabular}

Overall results from all subjects that completed the trial. No statistically- significant evident.

Abbreviations: $m L$ : milliliters, IQR: interquartile range, AE: subjects suffering adverse events

Table 3b. Results, hemithyroidectomies only.

\begin{tabular}{|c|c|c|c|c|c|}
\hline & & Placebo & Evicel & Test & Significance \\
\hline First 8hours output (mL) & Median & 30.0 (IQR:24.0-38.8) & 50.0 (IQR: 41.0-71.5) & Mann-Whitney & 0.105 \\
\hline Total output $(\mathrm{mL})$ & Median & 62.5 (IQR:53.3-108.6) & 90.5 (IQR: 67.8-149.4) & Mann-Whitney & 0.218 \\
\hline Drain time (hours) & Median & 32.4 (IQR: 24.8-40.8) & 30.5 (IQR: 25.8-40.0) & Mann-Whitney & 0.853 \\
\hline Hospital stay (hours) & Median & 39.9 (IQR: 26.1-46.0) & 29.8 (IQR: 28.6-33.4) & Mann-Whitney & 0.918 \\
\hline AEs & $\mathrm{n}, \%$ & $0,0 \%$ & $2,20 \%$ & Chi-square & 0.474 \\
\hline
\end{tabular}

Overall results from all subjects that completed the trial. No statistically- significant evident.

Abbreviations: $m L$ : milliliters, IQR: interquartile range, AE: subjects suffering adverse events

Table 3c. Results, total thyroidectomies only.

\begin{tabular}{|c|c|c|c|c|c|}
\hline & & Placebo & Evicel & Test & Significance \\
\hline First 8hours output (mL) & Median & 60.0 (IQR: $30.0-80.0$ ) & 50.0 (IQR: 32.8-64.4) & Mann-Whitney & 0.483 \\
\hline Total output $(\mathrm{mL})$ & Median & 150.0 (IQR: $120.0-188.5$ ) & 103.5 (IQR: 80.0-138.6) & Mann-Whitney & 0.035 \\
\hline Drain time (hours) & Median & 47.1(IQR: 42.0-67.8) & 40.3 (IQR: 26.2-49.1) & Mann-Whitney & 0.035 \\
\hline Hospital stay (hours) & Median & 59.4(IQR: 48.4-70.6) & 50.4 (IQR: 43.6-54.9) & Mann-Whitney & 0.246 \\
\hline AEs & $\mathrm{n}, \%$ & $3, \quad 17.6 \%$ & $7,38.9 \%$ & Chi-square & 0.264 \\
\hline
\end{tabular}

Results from total thyroidectomy subjects that completed the trial. No statistically- significant evident.

Abbreviations: $m L$ : milliliters, IQR: interquartile range, AE: subjects suffering adverse events

\section{Discussion}

This study shows a statistically significant improvement in two outcomes in the total thyroidectomy group - both total drainage and drain time were significantly reduced when using Evicel versus placebo, under a conservative drain management protocol. However, neither wound drainage in the first eight hours following surgery nor hospital stay were improved with Evicel. Evicel also had no significant impact on any study outcome in the hemithyroidectomy subgroup, or overall.

Since the fibrin sealant was only beneficial in total thyroidectomy, it may stand to reason that more extensive resections and larger potential spaces may stand to benefit the most from the use of Evicel in thyroid surgery. However, this study did not have sufficient power to demonstrate these supposed benefits in substernal goiter patients 
or neck dissection patients due to small sample size ( $\mathrm{N}=5$ for both). It would be interesting to see if a greater benefit continues to be demonstrated in these special cases, but such a study would likely require a multi-institutional trial in order to obtain sufficient sample size.

Two previous randomized, controlled studies [11,15] have investigated fibrin sealants in thyroid surgery. Kim et al. [11] studied their use for total thyroidectomy, with bilateral neck dissection, in 78 papillary thyroid cancer patients. Their results showed a borderline significant reduction in total drainage $(p=0.05)$ but demonstrated no difference in drain time, nor in hospital stay.

Uwiera et al., [15] studied the use of Tisseel (manufactured by Baxter International Inc., Deerfield, Illinois, USA) for both total and hemithyroidectomies, and showed a statistically-significant improvement in wound drainage overall and in both total and partial thyroidectomy subgroups, but did not demonstrate statisticallysignificant reductions in drain time, nor in hospital stay in any group.

This study contributes to a growing collection of literature that show that fibrin sealant is capable of reducing routine post-operative thyroid surgery drainage, but does not reduce drain time or hospital stay. However, considering the consensus against conservative drain protocols [3-5], it may be beneficial to study the use of fibrin sealant in "special" cases that do indicate drainage: where the goiter is massive or substernal, where the neck dissection is extensive, or in Graves' disease [7]. Conversely, it could also be studied in drainless outpatient hemiand total thyroidectomy in order to determine if its use reduces the rate of post-operative hematoma and seroma formation in this group. Outpatient surgery is becoming increasingly desirable for some patients undergoing partial or total thyroidectomy. The most recent consensus statement on this technique does not advocate for or against fibrin sealant [17], but use in this population may be worthy of additional investigation.

This study was not without limitations. The study was unsuccessful in accruing the planned study population in a timely manner, and so has only half the number of subjects that were originally planned. Two analyses had $p$-values $<0.15$ : overall drain time ( $p=0.101$, favoring Evicel) and hemithyroidectomy drainage in the first 8 hours $(\mathrm{p}=0.105$, favoring placebo). With a larger sample size, these comparisons may have demonstrated significance. Second, assisting surgeons were not blinded to the treatment group of the subjects, and actively participated in the care of the subjects at our teaching hospital. However, group allocation was never discussed openly during rounds or hand-offs, and residents attested readily that no treatment decisions were affected by group allocation.

\section{Conclusion}

This study contributes to a growing collection of literature that show that fibrin sealant is capable of reducing routine post-operative thyroid surgery drainage, but does not reduce drain time or hospital stay. Further study should investigate sealants for use in drainless thyroid surgery, or in more extensive surgical cases where drains are most likely to be used.

\section{Acknowledgements}

The authors would like to acknowledge the following personnel for their assistance in coordinating the study: Rishi Vashishta, Ezekiel Terrell, Wasef Muzzafar, Shivangi Lohia, P. Ryan Camilon, and the nursing staff in the PACU and $7^{\text {th }}$ floor inpatient unit at MUSC. This study was funded by Ethicon US, LLC, Somerville, New Jersey, USA. The sponsor had no role in data collection, analysis or the writing of this manuscript.

\section{Financial disclosures}

Ethicon, Inc. (Somerville, New Jersey, USA) funded this study and provided the Evicel ${ }^{\circ}$. The sponsor had no role in data collection, analysis or manuscript preparation.

\section{References}

1. Gharib H, Papini E, Paschke R, Duick DS, Valcavi R, et al. (2010) American Association of Clinical Endocrinologists, Associazione Medici Endocrinologi, and EuropeanThyroid Association Medical Guidelines for Clinical Practice for the Diagnosis and Management of Thyroid Nodules. Endocr Pract,. 16 Suppl 1:1-43. [Crossref]

2. Gourin CG, Eisele DW (2009) Complications of Thyroid Surgery, in Complications in Head and Neck Surgery, D.W. Eisele and R.V. Smith, Editors. 493-515.

3. Samraj K, Gurusamy KS (2007) Wound drains following thyroid surgery. Cochrane Database of Systematic Reviews. 4: CD006099.

4. Woods RS, Woods JF, Duignan ES, Timon C (2014) Systematic review and metaanalysis of wound drains after thyroid surgery. Br J Surg 101: 446-456. [Crossref]

5. Kennedy SA, Irvine RA, Westerberg BD, Zhang H (2008) Meta-analysis: prophylactic drainage and bleeding complications in thyroid surgery. Journal of OtolaryngologyHead and Neck Surgery. 37: 768-773. [Crossref]

6. Dunlap WW, Berg RL, Urquhart AC (2010) Thyroid drains and postoperative drainage. Otolaryngol Head Neck Surg 143: 235-238. [Crossref]

7. Minami S, Sakimura C, Hayashida N, Yamanouchi K, Kuroki T, et al. (2014) Timing of drainage tube removal after thyroid surgery: a retrospective study. Surg Today 44 : 137-141. [Crossref]

8. Matthew TL, Spotnitz WD, Kron IL, Daniel TM, Tribble CG, et al. (1990) Four years' experience with fibrin sealant in thoracic and cardiovascular surgery. Ann Thorac Surg 50: 40-43. [Crossref]

9. Ochsner MG, Maniscalco-Theberge ME, Champion HR (1990) Fibrin glue as a hemostatic agent in hepatic and splenic trauma. $J$ Trauma 30: 884-887. [Crossref]

10. Cain JE Jr, Dryer RF, Barton BR (1988) Evaluation of dural closure techniques. Suture methods, fibrin adhesive sealant, and cyanoacrylate polymer. Spine 13: 720-725. [Crossref]

11. Kim TW, Choi SY, Jang MS, Lee GG, Nam ME, et al. (2012) Efficacy of fibrin sealan for drainage reduction in total thyroidectomy with bilateral central neck dissection. Otolaryngol Head Neck Surg 147: 654-660. [Crossref]

12. Mohindra S, Mohindra S, Gupta K (2013) Endoscopic repair of CSF rhinorrhea: necessity of fibrin glue. Neurol India 61: 396-399. [Crossref]

13. Silberstein LE, Williams LJ, Hughlett MA, Magee DA, Weisman RA (1988) An autologous fibrinogen-based adhesive for use in otologic surgery. Transfusion 28: 319321. [Crossref]

14. Avery L (2014) Mann-Whitney U-test.

15. Uwiera TC, Uwiera RR, Seikaly H, Harris JR (2005) Tisseel and its effects on wound drainage post-thyroidectomy: prospective, randomized, blinded, controlled study. $J$ Otolaryngol 34: 374-378. [Crossref]

16. Schulz K, Altman D, Moher D (2010) CONSORT 2010 statement: updated guidelines for reporting parallel group randomised trials. BMJ 340: $\mathrm{c} 332$.

17. Terris DJ, Snyder S, Carneiro-Pla D, Inabnet WB 3rd, Kandil E, et al. (2013) American thyroid association statement on outpatient thyroidectomy. Thyroid 23: 1193-1202. [Crossref]

Copyright: (C)2016 Hornig JD. This is an open-access article distributed under the terms of the Creative Commons Attribution License, which permits unrestricted use, distribution, and reproduction in any medium, provided the original author and source are credited. 